\title{
Accessory brachial artery: a case report, embryological background and clinical relevance
}

\author{
David Kachlik ${ }^{\mathrm{a}}$, Marek Konarik ${ }^{\mathrm{a}}$, Miroslav Urban ${ }^{\mathrm{b}}$, Vaclav Baca $^{\mathrm{a}}$ \\ ${ }^{a}$ Department of Anatomy, Third Faculty of Medicine, ${ }^{b}$ Department of Histology, First Medical Faculty, \\ Charles University in Prague, Praha, 10000, Czech Republic
}

\begin{abstract}
Background: The accessory brachial artery (arteria brachialis accessoria) is a rare upper limb vascular abnormality, reported in less than one percent of cases. It is the artery originating from the axillary artery or the brachial artery, which rejoins the brachial artery further along its distal course within the arm or cubital fossa. Its detailed knowledge is necessary in transradial transulnar catheterization during coronary procedures, mainly due to its narrow caliber, which is responsible for the failure of the intervention performance.

Objectives: Present a case of uncharacteristic branching pattern of the accessory brachial artery.

Method: The case was observed during a routine dissection in the left axilla of a female cadaver at the Department of Anatomy at the Third Faculty of Medicine, Charles University in Prague.

Results: The infrapectoral part of the axillary artery gave rise to a branch that descended distally along the medial side of the arm. This artery accompanied firstly the ulnar nerve, then it diverted laterally towards the median nerve and coursed hidden behind it to re-enter the brachial artery within the distal part of the arm, next to the biceps brachii muscle. The calibre of the accessory brachial artery was two mm only.

Conclusion: The accessory brachial artery is a rare variant of the upper limb vascular system and its prevailingly narrow lumen can cause a failure of the transradial/transulnar catheterization intervention.
\end{abstract}

Keywords: Accessory brachial artery, anatomical variation, axillary artery, brachial artery, variant, variation

The accessory brachial artery (arteria brachialis accessoria, ABA) is a rare upper limb vascular abnormality, reported in less than one percent of cases $[1,2]$. ABA is the artery originating from the axillary artery (arteria axillaris, AA), or the brachial artery (arteria brachialis, BA) that rejoins the brachial artery further along its distal course. Its detailed knowledge is necessary particularly in transradial/ transulnar catheterization during coronary procedures, in surgery of the arm, and in cases of traumatic injuries.

The protocol for the research project has been approved by the Ethics Commission of the Third Faculty of Medicine, Charles University in Prague and it conforms to the provisions of the Declaration of Helsinki in 1995 (as revised in Edinburgh 2000). All human subjects gave informed consent and donors' anonymity has been preserved.

Correspondence to: David Kachlik, Department of Anatomy, Third Faculty of Medicine, Charles University in Prague, Rusk 87, Praha, 10000, Czech Republic. E-mail: david.kachlik @1f3.cuni.cz

\section{Report of the case}

During a routine dissection of a 62-year-old woman, we observed an unusual arterial branching pattern in her arm. We noticed a branch that arose from the infrapectoral (third part) of the axillary artery (pars infrapectoralis arteriae axillaris) and descended to the periphery to rejoin the BA at its terminus in the distal part of the arm. This artery, obviously the ABA, stemmed from the AA, approximately three $\mathrm{cm}$ distal to the origin of the subscapular artery (arteria subscapularis) and two $\mathrm{cm}$ distal to the lower margin of the pectoralis minor muscle (musculus pectoralis minor) as shown in Fig. 1. Its outer caliber at the origin was two $\mathrm{mm}$. The ABA stretched between two roots of the median nerve (radix medialis et lateralis nervi mediani), approximately three $\mathrm{cm}$ before they merged to form the trunk of the median nerve (i.e. it would have followed the regular course of the brachial superficial artery if it too had been present as a variant) (see Fig. 1). The artery was situated ventrally to the 
median nerve and then traveled medially, inside a connective tissue sheath along with the ulnar nerve on the medial side of the arm. It sent off a thick branch to supply the subcutaneous adipose tissue six $\mathrm{cm}$ distal to its origin. When descending, the artery tended to travel more laterally and as such diverted from the ulnar nerve towards the median one. In the lower part of the arm, the ABA sent off a thin muscular branch and finally rejoined the BA, hidden behind the median nerve (see Fig. 2). The list of the calibers of the aforementioned arteries can be seen in Table 1. The ABA was accompanied by two very thin veins along its course, both of which drained into the axillary vein (vena axillaris). The vascular pattern of the arteries contained in the forearm of this case corresponded to those of common textbook patterns, i.e. the BA bifurcated in the cubital fossa (fossa cubitalis) to give rise to the radial and ulnar arteries (arteria radialis et ulnaris).

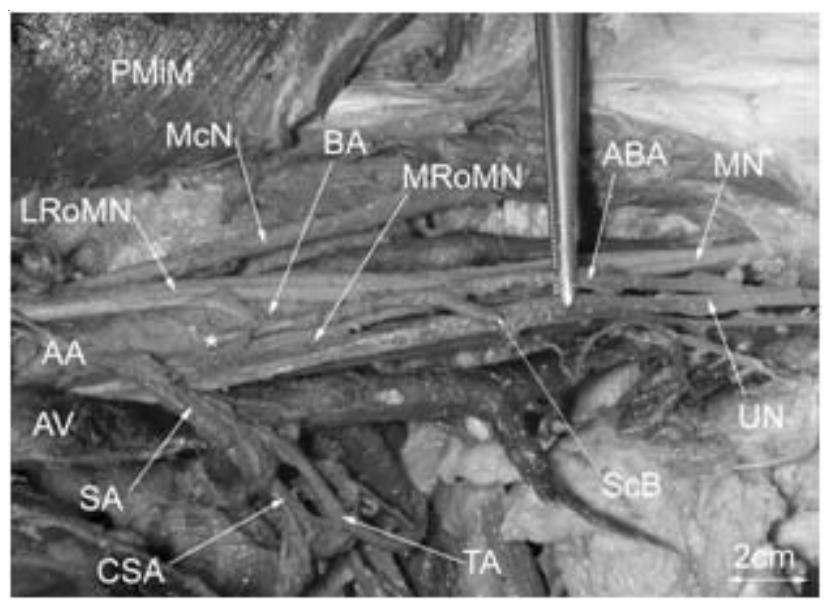

Fig. 1 Accessory brachial artery in the axilla and proximal arm. AA - axillary artery, ABA - accessory brachial artery, AV - axillary vein, BA - brachial artery, CSA - circumflex scapular artery, LRoMN - lateral root of median nerve, $\mathrm{McN}$ - musculocutaneous nerve, $\mathrm{MN}$ - median nerve, MRoMN - medial root of median nerve, PMiM - pectoralis minor muscle, SA - subscapular artery, ScB - subcutaneous branch, TA - thoracodorsal artery, UN - ulnar nerve, * - site of the origin of the accessory brachial artery

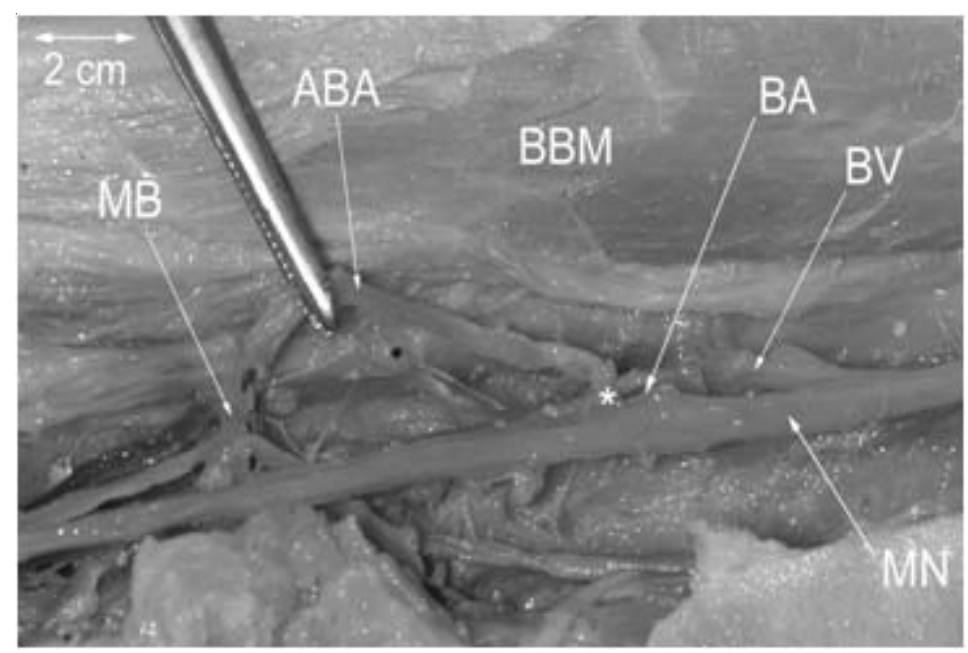

Fig. 2 Accessory brachial artery in the distal arm. ABA - accessory brachial artery, BA - brachial artery, BBM - biceps brachii muscle, BV - brachial vein, $\mathrm{MN}$ - median nerve, $\mathrm{MB}$ - muscular branch, * - site of the termination of accessory brachial artery 
Table 1. Calibers of arteries.

\begin{tabular}{ll}
\hline Artery & Caliber \\
\hline Axillary artery & $0.9 \mathrm{~cm}$ \\
Brachial artery & $0.7 \mathrm{~cm}$ \\
Accessory brachial artery & $0.2 \mathrm{~cm}$ \\
Subcutaneous branch & $0.15 \mathrm{~cm}$ \\
Muscular branch & $0.2 \mathrm{~cm}$ \\
\hline
\end{tabular}

\section{Discussion (Embryological background and clinical relevance)}

The term "accessory brachial artery" was first established by McCormack in 1953 [3]. Our case was observed during a larger study of more than 130 upper limbs. The embryological origin of such variations in the vasculature of the upper limb may be explained as an abnormal deviation in the normal vascular patterns. The proximal part of the right subclavian artery (arteria subclavia dextra) arises from the right aortic arch and the distal part of the artery is derived from the right seventh intersegmental artery (arteria intersegmentalis septima). The left subclavian artery has a different embryological background, however, as the entire artery is formed from the seventh intersegmental artery. The AA and its branches are derived from the axial artery, being a distal continuation of the seventh intersegmental artery on both sides of the body. Rodriguez-Baeza summarized the six main stages in upper limb development [4]:

26 Day: 2-3 $\mathrm{mm}$ bud of the future upper limb begins to form - the circulatory system of the bud can be characterized as a capillary network.

28 Day: 4-6 $\mathrm{mm}$ bud - the axial artery stems from the dorsal aorta (aorta dorsalis) and after it reaches the base of the limb, it begins to ramify. There are no structures developed at this stage.

33 Day: 7-9 mm bud - the axial trunk is developed as far as the AA. The peripheral vascular structures maintain the characteristic of a capillary network. The AA pierces the condensed nervous tissue and the nerves begin their migration towards the extremity.

41 Day: 11-14 mm bud - AA continues as the brachial artery, which reaches as far as the cubital fossa and gives off the deep brachial artery. The radial and ulnar nerves at this stage can be easily recognized. The humerus is chondrified, however the ulna and radius are represented by condense mesenchymal tissue only.
50 Day: $18-22 \mathrm{~mm}$ bud - all the structures of the upper limb begin to take adult characteristics and the nerve, skeletal and vascular patterns can be easily distinguished. The superficial brachial artery is defined and the distal part of the radial artery continues to retain the characteristic of a capillary network.

52 Day: $22-24 \mathrm{~mm}$ bud - the distal section of the radial artery is completely developed and the upper extremity now has a mature appearance.

The accessory brachial artery is derived from another embryological abnormality, referred to as the superficial brachial artery (arteria brachialis superficialis, SBA). The SBA is based on the persistence of more than one intersegmental cervical artery, which does not deteriorate but instead remains and can even grow larger, as stated by Jurjus et al. [5]. According to Adachi, the SBA is the brachial artery that travels superficially to the median nerve; this definition was altered somewhat by RodriguezNiedenfuhr who stated it runs "rather" superficially to the median nerve $[1,6]$. Obviously, this is because SBA often originates in an environment that makes it impossible to determine whether it is situated truly ventrally. This is the reason why such variations must be accompanied by accurate information as regards the branching originating from the infrapectoral part of the AA, the branches of the profunda brachii artery (arteria profunda brachii) and the branches of the SBA in the arm. Rodriguez-Niedenfuhr reported that in some cases, the artery travels dorsally to the median nerve [1]. The occurrence of the artery differs from $0.12 \%$ [3] and $0.2 \%$ [7] to $22 \%$ [ 8 ] and $25 \%$ finally [9]. The studies published over the previous 10 years (2000-2010) are far more precise, reporting the occurrence to be approximately 5\% (4.2\% Prasada Rao \& Chaudhary [10], 4.9\%, Rodriguez-Niedenfuehr [2], 5\% Kapur et al. [11], 5\% Konarik et al. [12], 6\% Patnaik et al. [13]).

The pattern of the ABA that we have presented in this article is much more uncommon - $0.77 \%$ according to our study. The first case that was ever reported in any literature concerning the ABA was published by Green in 1839 [14], and was followed by Quain in 1844 [7]. Ruge suggested denominating the ABA as the "Inselbildung" (island) because the appearance of stemming and rejoining resembles an island [15]. The fourth historical reference to this vascular variant was issued by Herrington who described a case of "high bifurcation and reunion of the brachial arteries" [16]. Quite interesting are 
Table 2 . The overview of studies concerning the accessory brachial artery.

\begin{tabular}{lc}
\hline Author & Percentage frequency \\
\hline Green $1830[14]$ & mentioned \\
Quain $1844[7]$ & $0.23 \%$ \\
Hazlett 1949 [21] & $0.53 \%$ \\
McCormack 1953 [3] & $0.13 \%$ \\
Keen 1961 [22] & $0.70 \%$ \\
Jurjus 1986 [23] & $0.70 \%$ \\
Rodriguez-Niedenfuhr 2001 [1] & $0.26 \%$ \\
Rodriguez-Niedenfuhr 2001* [2] & $0.70 \%$ \\
Kachlik 2010 & $0.77 \%$ \\
\hline
\end{tabular}

*Research made on embryos

Herrington's views on the system of variation of the AA. He proposed that some type of force favored bifurcation of the BA below the elbow. In the case of high origin of the BA, the same force favors the reunion of the branches demonstrated in the presented case. This statement is in contrast with new information regarding branching variations of the AA $[1,2,6]$, considering this type of variation as very rare as shown in Table 2.

McCormack was the first to use the term "accessory brachial artery" (considered as precise, short, and apposite), which was later applied by Rodriguez-Niedenfuhr in his thorough and comprehensive study [1-3]. We completely agree with Rodriguez-Niedenfuhr's terminology and believe that this clear, simple, and developmental approach should be followed. The terminology in the article follows the latest revision of the anatomical nomenclature as recommended by Kachlik et al. $[17,18]$.

The clinical relevance consists in the possible danger during the catheterization through the radial or ulnar artery, in the possibility of harvesting the radial artery as a graft or in the possible collateral circulation in cases of the arterial closure. The long axis of the ABA can concur with the disto-proximal axis of the radial artery and the catheter can enter the ABA. Its caliber is usually twice to thrice narrower than that of BA and does not allow the catheter to pass (or it evokes a heavy spasm). It is even thinner than the radial artery at its origin, which is reported 2.6 \pm 0.6 $\mathrm{mm}(1.6-3.8 \mathrm{~mm})$, measured two $\mathrm{cm}$ distal to the styloid process $[19,20]$. This accident can aggravate or even thwart the procedure. The ABA is more frequently hypoplastic or stenotic.

\section{Conclusion}

The accessory brachial artery (arteria brachialis accessoria) is a very rare vascular variation of the axillary artery, present in approximately $0.1-0.8 \%$ of cases. The anatomical knowledge of the brachial and antebrachial arterial pattern, as well as their possible variations and frequency, is crucial for many different medical fields, such as radiology, surgery (both reconstructive and plastic), oncology, intensive care units etc. The anomalous branch or branches of the axillary artery are a consequence of altered embryological conditions and can result in clinical problems such as heavy bleeding or poor perfusion of certain areas of the skin, subcutaneous tissue, muscles and in some situations even whole compartments. These serious complications may develop because of a routine injection in a hospital setting or in the privacy of a patient's home.

\section{Acknowledgment}

Special thanks to Mirka Plecita and Vladimir Musil for the technical and bibliographic support. The authors declare that they have no conflict of interest and no financial interests.

\section{References}

1. Rodriguez-Niedenfuhr M, Vazquez T, Nearn L, Ferreira B, Parkin I, Sanudo JR. Variations of the arterial pattern in the upper limb revisited: a morphological and statistical study, with a review of the literature. J Anat. 2001; 199:547-66.

2. Rodriguez-Niedenfuhr M, Burton GJ, Deu J, Sanudo JR. 2001. Development of the arterial pattern in the upper limb of staged human embryos: normal development and anatomic variations. J Anat. 2001; 
199:407-17.

3. McCormack LJ, Cauldwell EW, Anson BJ. Brachial and antebrachial arterial patterns: a study of 750 extremities. Surg Gynecol Obstet. 1953; 96:43-5.

4. Rodriguez-Baeza A, Nebot J, Ferreira B, Reina F, Perez J, Sanudo JR et al. Anatomical study and ontogenetic explanation of 23 cases with variations in the main patterns of the human brachio-antebrachial arteries. J Anat. 1995; 187:473-9.

5. Jurjus AR, Correa-De-Aruaujo R, Bohn RC. Bilateral double axillary artery: Embryological basis and clinical implications. Clin Anat. 1999; 12:135-40.

6. Adachi B. Das Arteriensystem der Japaner. Maruzen: Kyoto; 1928. p. 285-356.

7. Quain R. Anatomy of the Arteries of the Human Body. London: Taylor and Wolton; 1844. p. 326-37.

8. Lippert H, Pabst R. Arterial Variations in Man. J. F. Bergmann: M nchen; 1985. p. 71-3.

9. von Lanz T, Wachsmuth W: Praktische Anatomie vol. 1, part 3, Arm, 2nd ed. Berlin: Springer Verlag; 1959. p. 82-9.

10. Prasada Rao PVV, Chaudhary SC. Superficial brachial artery terminating as radial and superficial ulnar arteries. Cent Afr J Med. 2001; 467:78-80.

11. Kapur V, Suri RK, Manik P, Dhir V. Surgical anatomy of median nerve. J Anat Soc India. 2000; 49:92.

12. Konarik M, Knize J, Baca V, Kachlik D. Superficial brachioradial artery (the radial artery originating from the axillary artery): a case-report and its embryological background. Folia Morphol. (Warszawa). 2009; 68: 174-8.

13. Patnaik VVG, Kalsey G, Singla RK. Anomalous course of radial artery \& a variant of deep palmar arch - a case report. J Anat Soc India. 2000; 49:54-7.
14. Green PH. 1830. An Account of the Varieties in the Arterial System of the Human Body. Dublin: James Marshal Leckie; 1830. p. 16-20.

15. Ruge G. Beitr ge zur Gef sslehre des Menschen. Morph. Jahrb. 1884; 9:329-88.

16. Herrington M. High bifurcation of the brachial artery with reunion at the elbows. Johns Hopkins Hosp Bull. 1905; 16:65-6.

17. FCAT. Terminologia anatomica. Stuttgart: Thieme Verlag; 1998.

18. Kachlik D, Baca V, Bozdechova I, Cech P, Musil V. Anatomical Terminology and Nomenclature: Past, Presence and Highlights. Surg Rad Anat. 2008; 30: 459-66.

19. Yokoyama N, Takeshita S, Ochiai M, Koyama Y, Hoshino S, Isshiki $\mathrm{T}$ et al. Anatomic variations of the radial artery in patients undergoing transradial coronary interventions. Catheter Cardiovasc Interv. 2000; 49:357-62.

20. Yoo BS, Yoon J, Ko JY, Kim JY, Lee SH, Hwang SO et al. Anatomical consideration of the radial artery for transradial coronary procedures: arterial diameter, branching anomaly and vessel tortuosity. International J Cardiol. 2005; 101:421-7.

21. Hazlett JW. The superficial ulnar artery with reference to accidental intra-arterial injection. Can Med Assoc J. 1949; 61:289-93.

22. Keen JA. A study of the arterial variations in the limbs with special reference to symmetry of vascular patterns. Am J Anat. 1961; 108:245-61.

23. Jurjus A, Sfeir R, Bezirdjian R. Unusual variation of the arterial pattern of the human upper limb. Anat Rec. 1986;215:82-3. 